\title{
Factors Influencing Cloud Computing Adoption in Saudi Arabia's Private and Public Organizations: A Qualitative Evaluation
}

\author{
Mohammed Ateeq Alanezi \\ College of Computing and Information Technology \\ Shaqra University, Kingdom of Saudi Arabia
}

\begin{abstract}
Cloud Computing is becoming an important tool for improving productivity, efficiency and cost reduction. Hence, the advantages and potential benefits of cloud computing are no longer possible to be ignored by organizations. However, organizations must evaluate factors that influence their decisions before deciding to adopt cloud computing technologies. Many studies have investigated cloud computing adoption in developed countries compared with few studies that have concentrated on examining the factors that influence cloud computing adoption in developing countries. It is not clear to see whether these factors that have been identified by these studies, can be applied in developing countries. The motive of this study is to contribute to the adoption of cloud computing, and to elevate the consciousness of cloud computing technology amongst authorities, researchers, administrators, business enterprise managers and service carriers, particularly within the Saudi Arabian context. This study explores factors that encourage the implementation of cloud or have the capacity to detract from adopting cloud computing in private and public organizations in Saudi Arabia. A qualitative approach through IT professional representatives' interviews was adopted in this study, which explored two categories, namely, a) the negative impact category which includes: security and privacy, government policy, lack of knowledge, and Loss of control; and b) the positive impact category which includes three factors: reduce expenses, improve IT performance, and promote scalability and flexibility.
\end{abstract}

Keywords-Cloud computing; private and public organization adoption; qualitative evaluation; Saudi Arabia

\section{INTRODUCTION}

Organizations are, nowadays, changing their strategy in IT towards the adoption of cloud computing. The strategy is mainly connected with technological innovation, its flexibility in implementation and growth in economy. Hence it is not possible anymore for organizations to ignore the advantages of cloud computing [1]. Various areas such as businesses, institutions and government are attracted to cloud computing [2]. Cloud computing offers a shift from the current installed software in the personal computers to the cloud-based servers which can be accessed from anywhere at any time. In addition, cloud computing provides an on-demand provisioning of resources and scalability [3]. Various advantages such as business process transformation, real world applications delivery, lower expenditures in infrastructure, and mobilization are more likely to achieve higher efficiency in business strategy [4].
Cloud computing is attached to the scope of e-service as a potential means in order to serve the public and private sectors with more services [5]. It is a method of green technology that has a potential to improve utilization of the current resources in the data centre and lower their consumption. Energy savings in computing and storage service can be offered by the cloud computing through the use of large shared servers and storage units [6]-[8]. In order to realize these benefits, more information, development and promotion is needed to encourage the adoption [9]. The economic slump that hit recent years in IT has increased the interest of cloud services adoption, which can be used to reduce the costs [10]. There is a very limited number of theoretical studies that identified and evaluated the factors that impact the implementation of cloud computing. Most of the published work so far is white papers, newsletters and policy statements lacking academic methodology and analysis [3]. Most of these studies, which are empirical in nature, have focused only on the advantages and the associated risks of cloud computing [9]. A few studies have been found in the context of developing countries including Saudi Arabia [11]-[13]. Most of these studies have taken a well-established theory (such as Technology-Organization Environment framework (TOE), Diffusion of Innovation (DOl), and Technology Acceptance Model (TAM)) and collected data from a developing country in order to test if the theory or the model was applicable in that context. They failed to focus on the contextual details and the specific factors that are affecting the implementation of cloud computing in the local context. Thus, the main goal of this study is to identify the factors which influence the implementation of cloud computing in private and public organizations in Saudi Arabia.

\section{LITERATURE REVIEW}

Whenever the development takes place on a structural societal change in a place where various diverse social, and economic changes play a major role, it can be predicted that information and communication technology development is a very important factor [14]. Accessing the computing resources or developing robust IT infrastructure is a very difficult approach in developing countries. However, the new computer paradigms, such as cloud computing, had provided access to various resources that are available remotely or have been in any other case inaccessible. The evolution of cloud computing will change facing of this, which includes small and large-scale businesses, entrepreneurs, researchers and government systems [15]. However, it is not yet clear how the implication of cloud 
computing in public and private sectors will be handled. Still it can be predicted that information and communication technology have the potential to access the information that was not possible few years back. All the public and the private enterprises are trying to use the cloud-based services which is becoming increasingly important. As stated in [16], the concept of cloud computing is becoming the main strategy for various organizations. This short survey of latest literature and research findings on cloud computing is offered in three parts. The first part involves the conceptualization of cloud computing, the second part outlines its benefits, and the third part explores organizations' adoption of cloud computing.

\section{A. Overview of Cloud Computing}

Cloud computing can be defined as a technology paradigm that enables ubiquitous, appropriate, on-demand network access to share computing resources and services. Various computing resources like storage, servers, networks, applications and services which required to be rapidly provisioned and managed with minimal amount of efforts are offered in this paradigm [17]. Through the use of cloud computing technology, there is no need for users bother about managing resources and they get an access to all the available resources over the internet [1]. Cloud computing is defined as "a parallel and distributed computing system consisting of a collection of inter-connected and virtualized computers that are dynamically provisioned and presented as one or more unified computing resources based on Service-LevelAgreements (SLA) established through negotiation between the service provider and consumers" [18]. The service providers are trying to make software and technology available on demand. The computing and storage over the internet can be used by the end-user who does not require having the knowledge about the actual location and the configuration of the system, where his/her data is coming from and being saved.

Could computing provides three types of services [19], [20]. They include infrastructure as a service, Platform as a service and Software as a service.

- Infrastructure-as-a-Service (IaaS): In this service, the cloud offers some physical devices such as computers which are connected virtually, various servers, and devices used for storage as services. All these services are located in one physical place (data center) but they could be accessed only through the internet. The clients of cloud computing only pay for what resources and services they use. For example, organizations can use cloud computing technique in email management, website hosting, and data management.

- Platform-as-a-Service (PaaS): This service typically stands for computing platform. Through PaaS, developers can use all services over the cloud computing instead of purchasing it. In PaaS, licenses for various platforms such as all the operating systems, databases management, and other programming software, become easily available on the internet [11].

- Software-as-a-Service (SaaS): In SaaS, cloud providers maintain infrastructure and platform to provide applications in business such as word, emails, CRM, and ERP. All these apps are carried over the internet which can be available for the end-user. The end user can easily get an access to the apps anywhere at any time. This is the most utilized Cloud Model [11].

Free email services like Google and Microsoft are an example of a cloud computing approach that is already in action. However, there are various other services that can be used by private and public organizations which are launched recently that make use of cloud computing services such as data management, data analysis, mobile telecommunication services and various computing-based services that makes use of grid computing and parallel computing systems [21].

\section{B. Benefits of Cloud Computing}

In latest literature, it could be observed that challenges faced by organizations could be resolved by cloud computing which has been proved to be reliable [8]. The main advantages of cloud computing include offering lower expenses [22], better services standardization [23], and greater business adaptability [24]. Other advantages such as increased accessibility to cutting-edge technology and scalability of services, makes cloud computing technologies more appealing to be widely implemented in various sectors [6], [25]. Some of the other main advantages of cloud computing system are particularly in the application of e-government services which includes infrastructure minimization, improvement of network security, service scalability and implementation speed [26]. As suggested in [27], low total cost of ownership, low initial investment costs, faster time to benefits realization, continuous enhancement of the service offerings are usual benefits of implementing cloud computing. A study by Rath et al. [28] tried to find the advantages/benefits of the adoption of cloud computing by Indian companies. They concluded that the advantages for SMEs includes reduced investment in hardware, effective use of computing systems in current data centers, services and cost saving related to technology infrastructure and faster software upgrades with low expense.

\section{Cloud Computing Adoption in Organizations}

Many studies have been conducted on cloud computing adoption by private and public organizations. Neves et al. [29], have tried to identify various issues that are associated with social, economic, political and technological factors affecting the adaption of cloud computing and had given various ideologies to cope up with these issues. Makena [30] has identified the factors that has an effect on the adoption of cloud computing by Kenyan SMEs. Those factors are: technology, management support, relative advantage, size, preparedness, competitiveness, complexity and compatibility. Azarnik et al. [31] concluded that a risk-management program that is well-defined by which it focuses on cloud computing is essential for the success of organizations' cloud computing transformation. Services such as data mining, optimization, risk modeling and simulation which are computationally intensive are the most important benefits for cloud computing adoption in Indian organizations [28]. The support of software functionalities, the ubiquitous access to data, responsive solutions to customer support queries, maintaining huge data and reducing cost are key success factors for organization's customers to adopt cloud computing [32]. Stieninger and 
Nedbal [33] depicted that the factors influencing an implementation of cloud computing are perceived by safety and security, cost, trust management, and efficiency of energy. Hussein et al. [34] used qualitative and quantitative methods to investigate the awareness of cloud computing within SMEs present in Malaysia. As a result, the majority of the users only know about its benefits in terms of data backup and storage. A survey among seventeen IT managers was conducted in Spain concluded that they were also not aware of the cloud computing applications [35] .

From the earlier publications, it can be noticed that many articles which are published are using different theories and models to study the effective implementation of cloud computing. Technology-Organization-Environment framework (TOE) is used in [36] to investigate the effective implementation of cloud computing within the SMEs, especially in UK. Some researchers proposed the implementation of the cloud computing technology based on the already existing TOE framework as shown in [36], [38]. Furthermore, the theory of Diffusion Of Innovation (DOI) has also been used [33]. Some researchers have proposed the process of studying the implementation of cloud computing based on the already defined two models together in a combined form [33], [37]. For example, Saedi and Iahad [37] found that the Technology-Organization-Environment Framework (TOE) and Actor Network Theory (ANT) are suitable for examining cloud computing implementation in various SMEs. Furthermore, Technology Acceptance Model (TAM) applied among the various models and methodologies to study cloud computing adoption [39].

\section{METHODOLOGY}

This study has utilized a qualitative approach to identify factors that can impact cloud computing adoption. In this approach, IT professional representatives who were invited from various private and public organizations in Saudi Arabia were interviewed. In order to make sure that the sample reflects the geographical spread and size classifications of private and public organizations across Saudi Arabia, the selection of organizations across different industries in Saudi Arabia has been carried out. A summary of the profile of these organizations is presented in Table I.

TABLE I. ORganizations' PRofile

\begin{tabular}{|l|l|l|}
\hline Organization Domain & Organization type & No. of participants \\
\hline \multirow{2}{*}{ ICT } & Private & 3 \\
\cline { 2 - 3 } & Public & 4 \\
\hline Health & Private & 3 \\
\hline Construction & Private & 1 \\
\hline \multirow{2}{*}{ Manufacturing } & Public & 5 \\
\cline { 2 - 3 } & Private & 3 \\
\hline Education & Public & 4 \\
\hline Finance & Private & 3 \\
\hline Telecommunication & Private & 2 \\
\hline Traveling & Private & 1 \\
\hline Training centers & Private & 1 \\
\hline Research centers & Public & 2 \\
\hline
\end{tabular}

\section{A. Interview Guide and Process}

To improve the reliability of this research, only voluntary participants who were responsible for the planning and the cloud computing adoption in their organizations such as IT directors, IT practitioners, IT executive, IT managers, and system analysts were interviewed (Table II).

TABLE II. PARTICIPANTS' POSITION

\begin{tabular}{|l|l|}
\hline Participants' position & Quantity \\
\hline IT directors & 2 \\
\hline System analysts & 4 \\
\hline IT practitioners & 13 \\
\hline IT executive & 7 \\
\hline IT managers & 6 \\
\hline
\end{tabular}

An interview guide was developed and used to guide the interview method. In the guide, information about the study aim, rights of participant, responsibilities of the researcher and a confidentiality guarantee on the interview were provided. Interviewees were clearly notified that they may decline to answer any question and provided answers would not be classified as right or wrong (i.e. there is no right or wrong answers in this interview).

To explore the factors that impact cloud computing adoption, the semi-structured interviews incorporated a range of sub-questions through various themes (see appendix A). Pilot interviews with five academic staff in Shaqra University were conducted to examine the research questions' relevancy as well as to evaluate the guide that was given to interviewees. The pilot interviews led to modifications and improvements on the interview questions as well as the interview guide.

The interviews kicked off with a general question about cloud computing; this was followed by questions related to the factors which led to encourage or discourage the organization to adopt cloud computing. Moreover, interviewees were asked to list the criteria which used by them to evaluate the readiness of their organization to adopt cloud computing. Questions about their experiences (positive and negative) with cloud computing adoption were asked. However, the questions as described were posed to the participants who responded in such a manner that the semi-structured nature of the interviews proved beneficial for the researcher with the flexibility of asking further questions to avoid bias by eliciting an obvious response [40]. Few interviewees were re-contacted to confirm or elucidate information in order to validate data.

The 32 personal in-depth interviews were conducted in various locations in Saudi Arabia where it was convenient to the participants between July and September 2017. Since most of the participants speak English fluently, English language used during the interviews, which were between 35 and 50 minutes. Only one participant preferred to be interviewed in Arabic. The interviews were tape-recorded with the permission of all interviewees. The interviews were carried out until the point of data saturation was reached [41].

\section{B. Data Analysis}

The analysis of the interview data identified the factors that impact cloud computing and the manner of that impact. Content analysis was utilized in the study as it is suitable for 
analyzing different communication materials including "narrative responses, open-ended survey questions, interviews, focus groups, observations, printed media such as articles, books, or manuals" [42]. Using the steps suggested by [43] for a content analysis process, to draw out the relevant items, themes and categories, the data collected was analyzed (Fig. 1).

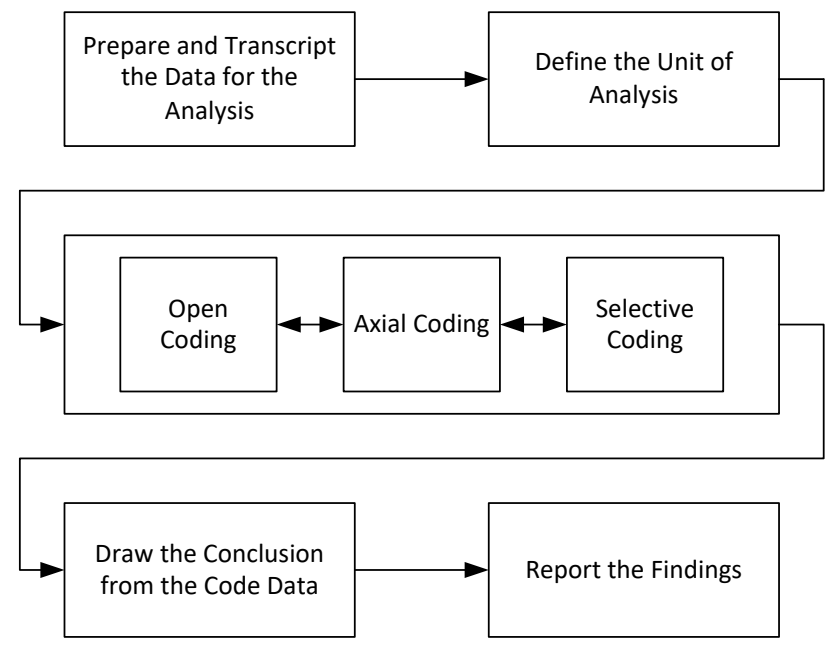

Fig. 1. Content analysis processes.

According to [43], usually, individual themes are utilized in qualitative content analysis as the unit for analysis; these themes might be articulated in a word, a phrase, a sentence, or a paragraph. Each interview was transcribed and prepared for data analysis. In this study, categories and a coding scheme were generated inductively from the data. In order to code qualitative data, the researcher has applied the three coding techniques proposed by [44] which are: open coding, axial coding and selective coding.

\section{1) Open Coding}

Open coding technique is used to identify discrete concepts and categories [44]. The open coding was accomplished by reading each transcript to note any interesting or relevant information by making a brief note in the page border about the nature of this information. From these border notes, the researcher made a list of codes from different types of information. After reading through the lists of open code that excerpted from transcripts, the researcher sorted and categorized these initial codes in the way that describe what they are about. Based on these processes, the researcher managed to generate 79 notes based on how different concepts are related and links.

\section{2) Axial Coding}

Axial coding is used to connect categories ( 79 concepts in the first stage) for the relationships by generating subcategories [44]. In this process all the initial concepts in open coding process were reduced by considering possible relationships with other concepts. Based on these processes, the initial 79 concepts were further refined and incorporated into 32 concepts. These 32 subcategories (concepts) were further classified into 7 categories (factors). These factors are security and privacy, lack of knowledge, government policy, loss of control reduce expenses, improve IT performance, and promote scalability and flexibility. The purpose of this process is to group together all subcategories with similar occurrences and incidents into categories. The concepts under each of the factors were then validated by scrutinizing possible relationships with other factors. This process was repeated for each factor to ensure the relationship is held. After repeating this process, all related factors from the transcribed interviews were saturated and no further relationships emerged. Finally, the original interview transcripts were reviewed to identify any possible outliers that could be incorporated into the factors. Further, direct quotes were extracted from the transcripts to support the qualitative data analysis findings [42].

\section{3) Selective Coding}

Selective coding is the third coding process which is used to discover the core categories. This stage was accomplished by integrating the related categories ( 7 factors in the axial coding stage) to discover the core-categories and refining them. The researcher linked these factors under two major categories such as positive impact and negative impact. The coding procedure begins with exploring concepts, followed by connecting the concepts for relationships under main factors, and finally connecting these factors to discover the corecategories. Fig. 2 was translated from the analysis processes to clarify the coding process.

The researcher compared the emerging theme from the data and the current concepts in cloud computing literature. Whenever consistency is founded, the researcher modified the concepts to match with the literature. For example, government policy was initially conceptualized in this study as regulation and law; however, after considering the same meaning concepts in the literature, this concept was modified to government policy. The researcher followed this way in order to be consistent with the literature and to add theoretical soundness to the conceptualization.

\section{RESUlT AND DiSCUSSION}

Cloud computing is a new technology which is implemented in the field of ICT. The effective adoption of cloud computing gradually changed the way of services presented by ICT. Many organizations have already started to realize the importance of implementing cloud computing more effectively [28]. Some of the organizations have also started to implement the technology [36]. However, various issues should be taken into consideration when implementing cloud computing technology. One of the interviewees (participant 16) answered a question about the meaning of cloud computing by replying "cloud computing provides an on-demand provisioning of resources and scalability. It is a form of IT services delivery over the internet. These services include servers, emails, storage, databases, networking, software, analytics and more". 


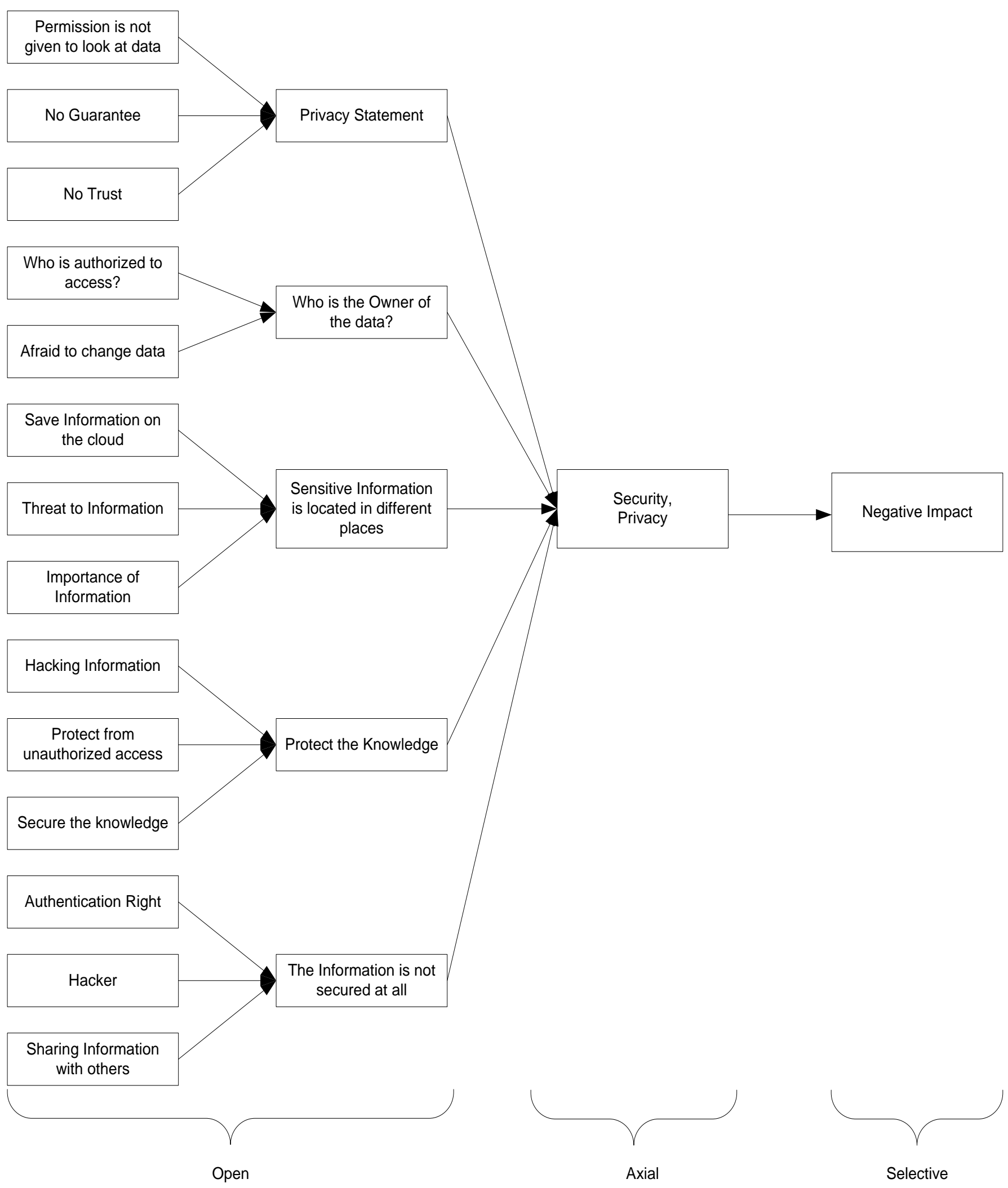

Fig. 2. Illustration of coding (privacy and security). 
In this study, the interviewees used different criteria to assess the factors that affect the adoption of cloud of computing. These criteria are sorted into two major categories and 7 factors as follows:

\section{A. Negative Impacts}

This category has the factors that hinder the adoption of cloud computing by organizations. The results revealed after analyzing the data show that cloud computing can be negatively affected by four factors as shown in Fig. 3.

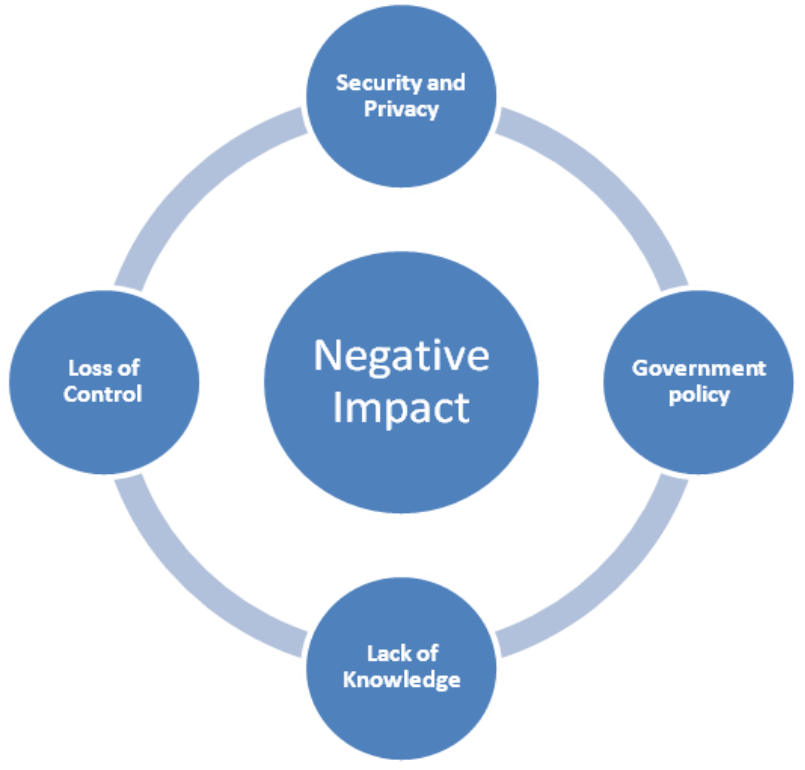

Fig. 3. Negative impact factors.

1) Security and privacy

In this study, security and privacy have been identified as one of the main factors impeding the adoption of cloud computing. Unsurprisingly, the same has been found in many previous research [31], [36]. As per [45], security and privacy is one of the key challenges that keep organization away from the adopting cloud computing. Most of the participants in this study saw the security and privacy as major obstacle in adopting cloud. One of the interviewees (participant 1) mentioned that "before we agree to shift to cloud technologies we need to ensure that our data is secured in the cloud and what the ability does the cloud provider have to deal with denial of service attacks". As cloud computing includes various technologies including databases, virtualization, resource scheduling, networks, operating systems, memory management, load balancing, concurrency control and transaction management. Consequently, security issues for many of these systems and technologies are applicable to cloud computing. This was confirmed by participant 9 who said "we need to understand the security requirements of the exit process. For instance, cloud service provider must allow secured network traffic and block malicious network traffic. Also, provider should give customers the essential tools to protect their system". Similar to this result, Seifu et al. [46] confirmed that the vulnerabilities and threats from infrastructure and network, up to the service platform was major concern in cloud computing adoption.
One of the major concerns is private leakage that has raised a great restriction for many organizations to execute cloud applications [47]. Likewise, many interviewees expressed their concerns on privacy as shown below:

"The secure processing of personal data and organizational data in the cloud computing represents a very big challenge" (participant 13).

"In my opinion, it is difficult to provide your data and business application to a third party because the security and privacy issues become a critical concern these days" (participant 21).

\section{2) Government policy}

Government policy issue has been seen from participants perspective as a threatening factor for the successful implementation of cloud computing. This is consistent with [22], [46]. Government policies regarding how cloud computing applications are imported and implemented can affect the decision of adopting cloud computing within an organization negatively [46]. The interviewees (participant 4 and 9) agree that the government policy related issues such as geographic location of systems and servers cannot be controlled. Furthermore, some governments-imposed restrictions on cross border data flows for privacy protection and security. One interviewee (participant 9) said that "Cloud computing has grown to be a new phenomenon used by every organization in Saudi Arabia. In this case the government definitely need to control and organize its use". Whereas as (participant 6) said that "there are some restricted policies by Saudi government to store the governmental data out of country".

\section{3) Lack of knowledge}

The rate of adoption of cloud computing is directly linked to levels of skills and expertise about cloud computing according to [30]. In supporting to this claim one of the interviewees (participant1) said that "lack of awareness is a key obstacle to cloud usage". Whereas, (participant 3) said "many businesses are not able to reap the full benefits of the cloud service because they do not fully understand what it provides". This is consistent with a study conducted by [1], [48] and concluded that lack of knowledge had a substantial effect on the attitude towards trust and the usage of cloud services. Additionally, the lack of standardization makes it more difficult to know if someone has the required knowledge. One of the interviewees (participant 30) confirmed that "different cloud services providers use different terminologies". Therefore, researchers and cloud computing agencies should play an important role to raise the level of cloud technology skills. Moreover, organizations also have to take time to train their employees if they want properly identified, utilize and expertise the service cloud computing.

\section{4) Loss of control}

Cloud service means that your data and applications are being hosted by someone else's server, not exclusively on your own server. One of the interviewees (participant 11) said "no one believes that cloud platforms as they exist today are perfect in controlling their data". To be fair, those who complain about lack of control are often thinking about direct hardware 
access. Another interviewee (participant 28) said "Loss of control over data: service user does not know where exactly its data is stored and processed in the cloud". However, many managers believe that access control is more problematic with cloud computing. In addition, the biggest issue is that the data travel across the internet and we have no control on neither physical infrastructure nor entry/exit outlet points [49].

\section{B. Positive Impact}

This category has the factors that encourage the adoption of cloud computing in organizations. The results revealed after analyzing the data show that cloud computing can be positively affected by three factors as shown in Fig. 4.

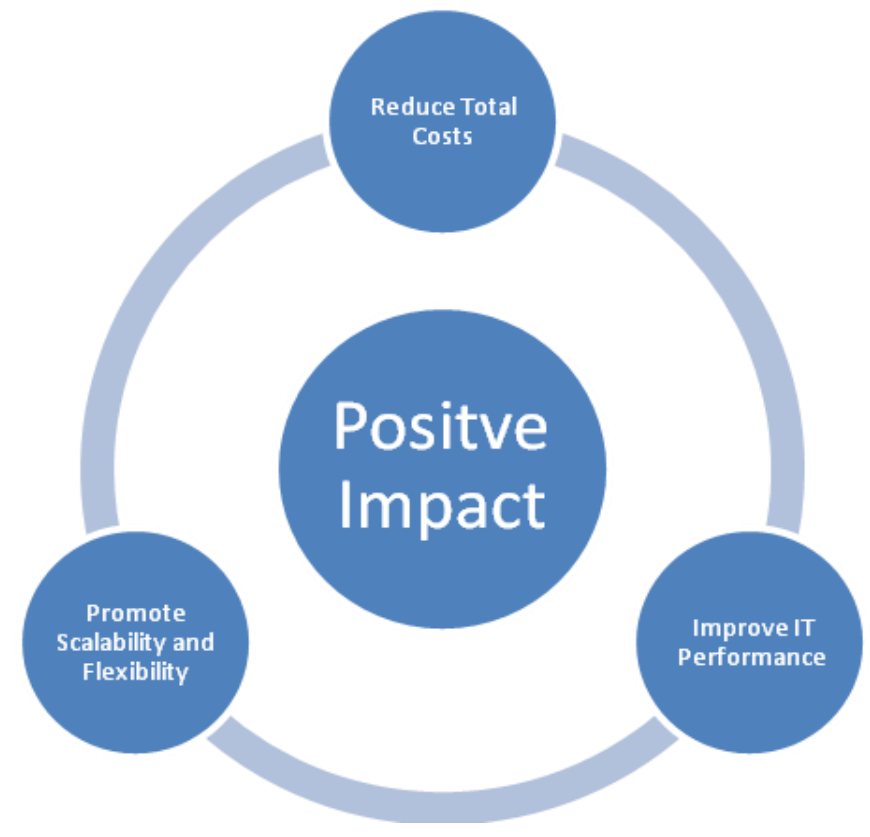

Fig. 4. Positive impact factors.

\section{1) Total cost reduction}

Reduction in total cost has been identified as one of the major factors that encourage organizations to adopt cloud computing. For most organizations, traditional process often involves buying technology equipment and tools, getting consulting services, paying for installation and license, and signing up to long maintenance contract. One interviewee (participant 21) mentioned that "with cloud services, there are no software licensing to purchase, no extra cost for installation services and no annual maintenance contract to renew". This is consistent with [50] who claimed that better effective use of cloud computing technology leads to cost savings and productivity gains. The key advantage of cloud computing is to increase the utilization compared to the process of traditional data centers, in term of the cost of electricity, bandwidth utilization, related infrastructure for operations, software, and hardware required. One of the interviewees (participant 13) mentioned "moving to cloud helps to have better resources and efficient bandwidth availability with less cost". In cloud computing, an organization pay based on the hour for computing resources, which leads to cost savings since the rate to rent a machine from a cloud provider is much lower than the cost to own one [22]. Thus, cloud computing offers services with minimum costs and maximize the profit in contrast to a data center [50].

\section{2) Improvement of IT performance}

There are several reasons that make cloud computing a beneficial tool for any organization. One of those reasons identified by this study is that cloud computing provides lesser cost computers to users. In other words, the user does not have to have a high-end computer to run cloud computing webbased applications since these applications run in the cloud instead of running them on a user computer. Therefore, cloud computing technologies improve the IT performance by sharing resources [10]. The interviewees agree that cloud computing provides low cost for IT infrastructure with the use of cloud computing recourses. Consequently, the IT department of an organization does not have to invest in a very large number of powerful servers, software and hardware resources. This helps the IT department to enhance or change the internal computing resources. This beneficial tool of cloud computing improves IT performance by increasing the throughput, avoiding load balancing issues, minimizing the delay and response time for transactions, since it is considered as a type of parallel, virtual, distributed, configurable, and flexible systems [50]. This is confirmed by an interviewee (participant 10) who mentioned that "when the organization needs to improve their performance, they should to think about adopting the cloud computing".

\section{3) Promote scalability and flexibility}

Scalability is one of the cloud computing performance measurements which is directly perceived by users as technical service reliability and availability, as well as by scalability of the applications [4]. Scalability is one of the important keys to the success of many organizations that are doing business in web applications. Furthermore, maintaining sufficient resources to meet the requirements might be costly [51]. This study discovered that most of the interviewees' participants agreed that promoting scalability and flexibility in any organization is the key factor of providing efficient services, which has a direct impact for capacity, reliability, and availability in cloud computing environment. Cloud computing enables organization employees to be more flexible by sharing documents and other online services, which can also help support internal and external collaboration. Furthermore, cloud computing allows the organization to upgrade or reduce IT requirements as needed. For example, most cloud service providers will allow organizations to increase their existing resources to accommodate increased business needs or changes. This will allow organizations to support their economy growth without making costly changes to their existing IT systems. One of the interviewees (participant 32 ) added that "one of the keys successful in adoption cloud computing is IT resource scalability which any organization are in need to provide high processing and large storage capabilities at the lowest possible cost".

\section{CONCLUSION AND FUTURE WORK}

There are many factors which need to be considered before adopting cloud computing by private and public organizations in Saudi Arabia. The research findings show some important 
issues that are influencing the adoption of cloud computing by these organizations. Security and privacy is one of the top leading issues in determining the decision for adopting cloud computing in these organizations. The vulnerabilities and threats from infrastructure and network was a major concern in adopting such technology. This research also shows that an increase in the awareness of cloud computing among private and public organizations serves as the perfect recipe for increased adoption and acceptance of this technology. The Saudi government shall play important role in determining which areas of improvement must be carried out to encourage the spread of ICT and cloud-based solutions. The results depicted that there are some restricted policies by Saudi government to store the governmental data out of country. Therefore, it is recommended that the Saudi government should adopt a new regulatory strategy that will promote the new technology of cloud computing and its impact on security, privacy, and personal data protection. Furthermore, the potential finding of this research illustrates that by adopting cloud computing technologies, IT performance in organizations can be improved, since it provides low cost for IT infrastructure with the use of cloud computing recourses.

The findings of this research recommend that strategies aimed at upgrading the regulatory approach should be introduced. Furthermore, launching this should be strongly advocated in the interests of Saudi Arabia to face the challenges of a successful migration to cloud computing while maintaining international standards. This study finds that mangers and cloud services providers should invest considerably greater resources in adopting cloud computing technologies. Aspects for consideration include improving security and ensuring the privacy are mandatory to guarantee a safe environment for organizations to adopt cloud computing technologies.

In light of the research outcomes, the factors identified are now being tested quantitatively and will be reported in due course. Using this data in various environments and contexts to provide cross-cultural comparisons may enrich the literature and result in understanding the adoption of cloud computing. Also future research can investigate whether the organizations' characteristics affect the adoption of cloud computing.

\section{ACKNOWLEDGMENT}

The author would like to thank Shaqra University, Saudi Arabia for providing a great research environment with excellent infrastructure. The author would also like to thank all participants who have attended the interviews.

\section{REFERENCES}

[1] Armbrust, M., et al., A view of cloud computing. Communications of the ACM, 2010. 53(4): p. 50-58.

[2] Buyya, R., et al., Cloud computing and emerging IT platforms: Vision, hype, and reality for delivering computing as the 5th utility. Future Generation computer systems, 2009. 25(6): p. 599-616.

[3] Ali, O., et al. Collaborative cloud computing adoption in Australian regional municipal government: An exploratory study. in Computer Supported Cooperative Work in Design (CSCWD), 2015 IEEE 19th International Conference on. 2015: IEEE.

[4] Low, C., Y. Chen, and M. Wu, Understanding the determinants of cloud computing adoption. Industrial management \& data systems, 2011. 111(7): p. 1006-1023.
[5] Sabi, H.M., et al., Conceptualizing a model for adoption of cloud computing in education. International Journal of Information Management, 2016. 36(2): p. 183-191.

[6] Lin, A. and N.-C. Chen, Cloud computing as an innovation: Percepetion, attitude, and adoption. International Journal of Information Management, 2012. 32(6): p. 533-540.

[7] Wang, C., et al. Privacy-preserving public auditing for data storage security in cloud computing. in Infocom, 2010 proceedings ieee. 2010: Ieee.

[8] Avram, M.-G., Advantages and challenges of adopting cloud computing from an enterprise perspective. Procedia Technology, 2014. 12: p. 529534.

[9] Rittinghouse, J.W. and J.F. Ransome, Cloud computing: implementation, management, and security. 2016: CRC press.

[10] Nikkhah, H.R. and R. Sabherwal, Mobile Cloud-Computing Applications: A Privacy Cost-Benefit Model. 2017.

[11] Alkawsi, G.A., A.K. Mahmood, and Y.M. Baashar. Factors influencing the adoption of cloud computing in SME: A systematic review. in Mathematical Sciences and Computing Research (iSMSC), International Symposium on. 2015: IEEE.

[12] Alhammadi, A., C. Stanier, and A. Eardley, The Determinants of Cloud Computing Adoption in Saudi Arabia. Computer Science \& Information Technology, 2015. 55.

[13] Al-Ruithe, M., E. Benkhelifa, and K. Hameed, Current State of Cloud Computing Adoption-An Empirical Study in Major Public Sector Organizations of Saudi Arabia (KSA). Procedia Computer Science, 2017. 110: p. 378-385.

[14] Sumner, A., M.A. Tribe, and M.A. Tribe, International development studies: Theories and methods in research and practice. 2008: Sage Los Angeles, CA.

[15] Greengard, S., Cloud computing and developing nations. Commun. ACM, 2010. 53(5): p. 18-20.

[16] Dahiru, A.A., J.M. Bass, and I.K. Allison, Cloud computing: adoption issues for sub-Saharan African SMEs. 2014

[17] Mell, P. and T. Grance, The NIST definition of cloud computing. 2011.

[18] Voorsluys, W., J. Broberg, and R. Buyya, Introduction to cloud computing. Cloud computing: Principles and paradigms, 2011: p. 1-41.

[19] Gupta, P., A. Seetharaman, and J.R. Raj, The usage and adoption of cloud computing by small and medium businesses. International Journal of Information Management, 2013. 33(5): p. 861-874.

[20] Malligai, V. and V.V. Kumar, Cloud Based Mobile Data Storage Application System. International Journal of Advanced Research in Computer Science and Technology, 2014. 2: p. 126-128.

[21] McLeod, C., Cloud Computing Service for SMEs Launched in Uganda. 2013.

[22] Saeed, I., G. Juell-Skielse, and E. Uppström, Cloud enterprise resource planning adoption: Motives \& barriers. Advances in Enterprise Information Systems II, 2012. 429.

[23] Aljabre, A., Cloud computing for increased business value. International Journal of Business and social science, 2012. 3(1).

[24] Sether, A., Cloud Computing Benefits. Browser Download This Paper, 2016.

[25] Miller, M., Cloud computing: Web-based applications that change the way you work and collaborate online. 2008: Que publishing.

[26] Greer Jr, M.B., Software as a service inflection point: Using cloud computing to achieve business agility. 2009: iUniverse.

[27] Seethamraju, R. Determinants of SaaS ERP Systems Adoption. in PACIS. 2013.

[28] Rath, A., et al. Decision points for adoption cloud computing in small, medium enterprises (SMEs). in Internet Technology And Secured Transactions, 2012 International Conference for. 2012: IEEE.

[29] Neves, F.T., et al., The adoption of cloud computing by SMEs: identifying and coping with external factors. 2011.

[30] Makena, J.N., Factors that affect cloud computing adoption by small and medium enterprises in Kenya. International Journal of Computer Applications Technology and Research, 2013. 2(5): p. 517>< meta name $=$. 
[31] Azarnik, A., et al., Associated risks of cloud computing for SMEs. Open International Journal of Informatics, 2012. 1(1): p. 37-45.

[32] Agostino, A., K.S. Søilen, and B. Gerritsen, Cloud solution in Business Intelligence for SMEs-vendor and customer perspectives. Journal of Intelligence Studies in Business, 2013. 3(3).

[33] Stieninger, M. and D. Nedbal. Diffusion and acceptance of cloud computing in SMEs: towards a valence model of relevant factors. in System Sciences (HICSS), 2014 47th Hawaii International Conference on. 2014: IEEE.

[34] Hussein, W.N., R. Sulaiman, and A.K. Hamzah. E-business and cloud computing awareness for Malaysian SMEs: A recommendation from academic and industry perspectives. in Research and Innovation in Information Systems (ICRIIS), 2013 International Conference on. 2013: IEEE.

[35] Trigueros-Preciado, S., D. Pérez-González, and P. Solana-González, Cloud computing in industrial SMEs: identification of the barriers to its adoption and effects of its application. Electronic Markets, 2013. 23(2): p. 105-114.

[36] Alshamaila, Y., S. Papagiannidis, and F. Li, Cloud computing adoption by SMEs in the north east of England: A multi-perspective framework. Journal of Enterprise Information Management, 2013. 26(3): p. 250-275.

[37] Saedi, A. and N.A. Iahad. Developing an instrument for Cloud Computing adoption by Small and Medium-sized Enterprises. in Research and Innovation in Information Systems (ICRIIS), 2013 International Conference on. 2013: IEEE.

[38] Kihara, T. and D. Gichoya. Adoption and use of cloud computing in small and medium enterprises in Kenya. in IST-Africa Conference and Exhibition (IST-Africa), 2013. 2013: IEEE.

[39] Tjikongo, R. and W. Uys. The viability of cloud computing adoption in SMME's in Namibia. in IST-Africa Conference and Exhibition (ISTAfrica), 2013. 2013: IEEE.

[40] Easterby-Smith, M., R. Thorpe, and P.R. Jackson, Management research. 2012: Sage.

[41] Guest, G., A. Bunce, and L. Johnson, How many interviews are enough? An experiment with data saturation and variability. Field methods, 2006. 18(1): p. 59-82.

[42] Hsieh, H.-F. and S.E. Shannon, Three approaches to qualitative content analysis. Qualitative health research, 2005. 15(9): p. 1277-1288.

[43] Zhang, Y. and B. Wildemuth, Qualitative analysis of content. 2009. USA: Libraries Unlimited Inc.

[44] Strauss, A.L. and J. Corbin, Pesquisa qualitativa: técnicas e procedimentos para o desenvolvimento de teoria fundamentada. 2008: Artmed.

[45] Schneiderman, R., For Cloud Computing, the Sky Is the Limit [Special Reports]. IEEE Signal Processing Magazine, 2011. 28(1): p. 15-144.
[46] Seifu, S.D., et al., Cloud-Computing: Adoption Issues for Ethiopian Public and Private Enterprises. The Electronic Journal of Information Systems in Developing Countries, 2017. 78(1): p. 1-14.

[47] Qiu, M., et al., Privacy-preserving smart data storage for financial industry in cloud computing. Concurrency and Computation: Practice and Experience, 2018. 30(5).

[48] Despotović-Zrakić, M., V. Milutinović, and A. Belić, Handbook of research on high performance and cloud computing in scientific research and education. 2014: IGI Global.

[49] Ahmed, A.A. and C. Xue Li, Analyzing data remnant remains on user devices to determine probative artifacts in cloud environment. Journal of forensic sciences, 2018. 63(1): p. 112-121.

[50] Chieu, T.C., et al. Dynamic scaling of web applications in a virtualized cloud computing environment. in E-Business Engineering, 2009. ICEBE'09. IEEE International Conference on. 2009: IEEE.

[51] Subashini, S. and V. Kavitha, A survey on security issues in service delivery models of cloud computing. Journal of network and computer applications, 2011. 34(1): p. 1-11.

\section{APPENDIX A}

Interview Questions:

1) What does the cloud computing technologies mean for you?

2) Do you use any of these technologies before?

3) If yes, can you describe of these technologies and how it was deployed and how it was delivered to you via the internet?

4) If no, why did you not use these technologies ?

5) How secure do you think of the use of cloud computing technologies?

6) What specific criteria did you use to evaluate the readiness of your organization to adopt cloud computing ?

7) What aspects of adoption of cloud technologies led you to positive experiences?

8) What aspects of adoption of cloud technologies led you to negative experiences?

9) From your experience, what factors that will encourage organization to adopt cloud computing?

10) From your experience, what factors that will discourage organization to adopt cloud computing?

11) Can you describe the challenges, risks, privacy and trust issues associated with cloud computing adoption? 